\title{
Modelle ressourcenorientierter und effektiver Führung digitaler Projekt- und Teamarbeit - vLead
}

\author{
Conny H. Antoni, Erich Latniak und Ulrike Hellert
}

\subsection{Zielsetzung und Vorgehen}

Gesamtziel des Vorhabens vLead war die Erforschung, partizipative Entwicklung, Praxiserprobung und Verbreitung von Konzepten und Instrumenten zur Unterstützung ressourcenorientierter und effektiver Führung und Gestaltung digitaler Projekt- und Teamarbeit. Diese sollen auch über das Projekt hinaus insbesondere KMUs in unterschiedlichen Branchen zur Verfügung stehen und sie bei der Digitalisierung ihrer Arbeits- und Geschäftsprozesse und bei der Entwicklung der dazu erforderlichen Kompetenzen ihrer Belegschaft unterstützen. Zur Erreichung dieser Ziele wurde

Unser Dank gilt für die Mitarbeit an dem Beitrag Valeria Bernardy, Rebecca Müller und Anna T. Röltgen (ABO-Psychologie, Universität Trier), Jennifer Schäfer (IAQ, Universität Duisburg-Essen) sowie Rebekka Mander und Frank Müller (iap, FOM Hochschule für Oekonomie \& Management $\mathrm{gGmbH}$ ), sowie für die Mitarbeit im Projekt Matthias Quinten (Data One GmbH), Karl-Friedrich Brockhaus und Rainer Trieb (Human Solutions Verwaltungs $\mathrm{GmbH}$ ) sowie beteiligten Kolleginnen und Kollegen in den jeweiligen Projektpartnerunternehmen. Für die Unterstützung im Projekt seitens des Projektträgers Karlsruhe PTKA bedanken wir uns ganz herzlich bei Stefan Scherr.

\section{H. Antoni $(\bowtie)$}

Universität Trier, Abteilung für ABO-Psychologie, Trier, Deutschland

E. Latniak

Universität Duisburg-Essen, Institut Arbeit und Qualifikation,

Duisburg, Deutschland

U. Hellert

FOM Hochschule, iap Institut für Arbeit \& Personal,

Nürnberg, Deutschland 
zunächst die Ausgangssituation in den Unternehmen der Projekt- und Umsetzungspartner analysiert. Es wurden die Anforderungen und Ressourcen digitaler Führung und Teamarbeit sowie ihre kulturellen und strukturellen Rahmenbedingungen erfasst. Gemeinsam mit den beteiligten Unternehmen wurden Konzepte und Instrumente für digitale Führung und Team-/Projektarbeit für verschiedene Handlungsfelder auf Basis dieser Erkenntnisse entwickelt und erprobt. Die partizipativ erarbeiteten Instrumente und Konzepte für Umsetzungsmaßnahmen unterstützen Unternehmen, operative Führungskräfte und (Projekt-)Teams bei der Gestaltung effektiver und gesundheitsförderlicher digitaler Projekt- und Teamarbeit. Sie geben insbesondere KMUs Hilfestellungen den digitalen Wandel der Arbeit zu bewältigen und dabei Unternehmens- und Beschäftigteninteressen, wie z. B. nach größerer Flexibilität und besserer Work-Life-Balance, zu vereinbaren.

Ziel des Teilprojekts der ABO war die Beantwortung der Frage, wie Führung in einer digitalisierten Arbeitswelt Einfluss nehmen kann, um Innovations- und Leistungsfähigkeit digitaler Projekt- und Teamarbeit sowie Work-Life-Balance und Gesundheit der Teammitglieder zu fördern. Es sollten wissenschaftlich fundierte und praxiserprobte Führungsinstrumente bereitgestellt werden, die insbesondere auf die Bedürfnisse von KMUs angepasst sind. Die partizipative Entwicklung und Erprobung dieser online basierten Führungsinstrumente erfolgte in enger Abstimmung mit den Projektpartnern Data One GmbH und der Human Solutions Verwaltungs GmbH sowie weiteren Unternehmen als Umsetzungspartnern. Ziel des Teilprojekts der Human Solutions Verwaltungs $\mathrm{GmbH}$ war die Entwicklung des Innovationsmanagement Tools IdeaCheck zur digitalen Unterstützung und Führung von Innovationsprozessen. Ziel des Teilprojekts der Data One GmbH war die Entwicklung des OrgaCheck als Screeningstool auf Unternehmensebene und des TeamCheck als Prozessmonitoring-Tool auf (Projekt-)Teamebene, um Belastungen und Ressourcen digitaler Führung und Zusammenarbeit zu analysieren. Hierzu wurden zunächst Situations- und Anforderungsanalysen mittels qualitativer und quantitativer Umfragen bei Führungskräften und deren Teammitgliedern durchgeführt. Anforderungen, Belastungsschwerpunkte und Ressourcen digitaler Führungs- und Teamarbeit wurden identifiziert. Ebenso wurden deren Auswirkungen auf individueller, sozialer und organisationaler Ebene in Abhängigkeit förderlicher und hinderlicher Rahmenbedingungen und Handlungsstrategien erfasst. Auf Basis dieser Ergebnisse wurden die Instrumente zur Unterstützung digitaler Führung und Teamarbeit gemeinsam entwickelt, erprobt und evaluiert. Die Toolentwicklung und -implementierung im Unternehmen wurde durch Interviews, Beobachtungen und quantitative Umfragen auf Basis des Technologie-Akzeptanzmodells (Unified Theory of Acceptance and Use of Technology, UTAUT) formativ evaluiert, vgl. [12].

Ziel des Teilprojekts des IAQ war zum Erhalt der Leistungsfähigkeit und Gesundheit virtuell arbeitender operativer Führungskräfte (opFk) beizutragen. Bedingt durch die variierenden Aufgaben und Verantwortlichkeiten entstehen bei opFk spezifische individuelle Anforderungen sowie Belastungs- und Ressourcenprofile. Zu deren Bewältigung entwickeln sie individuell unterschiedliche Strategien und Präferenzen. 
$\mathrm{Zu}$ deren Erfassung sollte ein - an die Bedarfe dieser Zielgruppe - angepasstes Vorgehen zur Reflexion der Arbeitssituation und zur Erarbeitung von Gestaltungs- und Regenerationsmaßnahmen entwickelt, sowie erfolgreiche Vorgehensweisen der Teilnehmenden dokumentiert werden. Zur empirischen Fundierung wurden zunächst eine Literaturrecherche und teilstandardisierte Interviews mit 13 Personen (u. a. mit opFk, Betriebsräten, Personalbereich) in zwei Unternehmen durchgeführt. Aufbauend auf diesen Befunden wurde ein Screening-Fragebogen entwickelt. Dieser erfasst Rahmenbedingungen, spezifische Belastungen (z. B. Informationsüberflutung, Zeitdruck), Ressourcen (z. B. Unterstützung durch die Führungskraft, berufliche Sinnerfüllung), individuelles Bewältigungs- und Coping-Verhalten (z. B. Nutzung von Kurzpausen), sowie ausgewählte Beanspruchungs- und Gesundheitsindikatoren (u. a. zur arbeitsbedingten Motivierung, Stress und Burnout). Insgesamt sollten so Bausteine für ein Coaching bzw. für die individuelle Arbeitsgestaltung von virtuellen opFk erarbeitet werden.

Ziel des Teilprojekts des iap war es, am Beispiel opFk und der von ihnen geführten Teams die Bedeutung der Zeitkompetenz in digitaler Projekt- und Teamarbeit und Führung zu untersuchen. Mit den Umsetzungspartnern wurde das Handbuch Kompass - Zeit und Vertrauen partizipativ entwickelt, erprobt und evaluiert: Er beinhaltet Instrumente und Strategien zur Förderung individueller und organisationaler Zeitkompetenz, Vertrauens- sowie Rückmeldestrukturen und -kulturen, die für wirksame und faire Entscheidungen benötigt werden. Um die Zeitkontrollmechanismen, Handlungsspielräume und vorhandenen Zeitkompetenzen der Führungskräfte und Beschäftigten sowie deren Strategien zum Erkennen und Lösen von Zeitproblemen zu identifizieren, wurden leitfadengestützte Interviews, Gruppendiskussionen, eine Beobachtung mit Führungskräften und Beschäftigten sowie im Anschluss eine standardisierte Onlineumfrage bei den Beschäftigten der Umsetzungspartner durchgeführt, vgl. [7].

\subsection{Forschungsergebnisse und Anwendungsbeispiele}

\subsubsection{OrgaCheck}

Die Ergebnisse der Situations- und Anforderungsanalyse zeigen, dass bei der digitalen Zusammenarbeit Belastungen und Ressourcen auf Personen-, Technik- und Organisationsebene unterschieden werden können. Hierzu gehören auf Personenebene beispielsweise die Entgrenzung von Arbeit und Freizeit sowie die Überlastung durch digital vermittelte Nachrichten, die zum Teil schwieriger zu interpretieren und einzuordnen sind als persönliche Kommunikation. Digitale Zusammenarbeit stellt dadurch erhöhte Anforderungen an die Selbstorganisation und Kompetenz im Umgang mit den unterschiedlichen digitalen Medien. Auf Technikebene geht es vor allem um zuverlässig funktionierende Hard- und Software. Auf der Organisationsebene stehen Regeln für die 
digitale Kommunikation und Zusammenarbeit und die Frage, wie die Führung digitale Zusammenarbeit unterstützen kann, im Fokus.

Der OrgaCheck ist ein online Umfrage-Tool, mit dessen Hilfe Herausforderungen und Ressourcen der digitalen Zusammenarbeit in Unternehmen auf Personen-, Technikund Organisationsebene analysiert werden können. Die Ergebnisse stehen automatisiert unmittelbar nach der Umfrage zur Verfügung. Der OrgaCheck zeigt Handlungsmöglichkeiten für Verbesserungen auf und kann somit Innovations- und Leistungsfähigkeit, sowie die Gesundheit von Führungskräften und Beschäftigten fördern. Zielgruppe sind alle Beschäftigten des Unternehmens. Die Unternehmensleitung setzt auf Basis der hinterlegten Fragen eine Onlineumfrage auf und lädt alle Beschäftigten zur Teilnahme ein. Der OrgaCheck unterscheidet drei Ebenen der Rückmeldung: Mitarbeitende erhalten ein individualisiertes Feedback zu wichtigen Aspekten digitaler Zusammenarbeit mitsamt Handlungsempfehlungen, siehe Abb. 13.1. Führungskräfte erhalten eine differenzierte Analyse, um Handlungsbedarfe innerhalb ihrer Abteilung zu identifizieren. Die Unternehmensführung erhält eine Rückmeldung mit Empfehlungen zu

\begin{tabular}{|c|c|c|}
\hline Individuelle Rückmeldung & Abteilungsrückmeldung & Untemehmensrückmeldung \\
\hline \multirow{2}{*}{ Indikatoren } & \multicolumn{2}{|c|}{ Beurteilung } \\
\hline & Schlecht & Gut \\
\hline \multicolumn{3}{|l|}{$\begin{array}{l}\text { Wahmehmung digitaler } \\
\text { Zusammenarbeit }\end{array}$} \\
\hline \multicolumn{3}{|l|}{$\begin{array}{l}\text { Belastung durch } \\
\text { Information }\end{array}$} \\
\hline \multicolumn{3}{|l|}{ Zufriedenheit } \\
\hline \multicolumn{3}{|l|}{ Wohlbefinden } \\
\hline \multicolumn{3}{|l|}{$\begin{array}{l}\text { Nützlichkeit der digitalen } \\
\text { Medien }\end{array}$} \\
\hline \multicolumn{3}{|l|}{$\begin{array}{l}\text { Effektivität digitaler } \\
\text { Arbeit }\end{array}$} \\
\hline $\begin{array}{l}\text { Identifikation mit dem } \\
\text { Unternehmen }\end{array}$ & & \\
\hline
\end{tabular}

Abb. 13.1 Beispielhafte Rückmeldung im OrgaCheck auf individueller Ebene 
Verbesserungen bezüglich Risiken und Ressourcen digitaler Zusammenarbeit für die langfristige Förderung der Leistungsfähigkeit und der Gesundheit von Führungskräften und Beschäftigten.

Folgende Aspekte werden zur Beurteilung der Herausforderungen und Ressourcen der digitalen Zusammenarbeit auf Personen-, Technik und Organisationsebene analysiert.

Auf Personenebene:

- Digitale Kompetenz: inwiefern sich Personen über digitale Medien ausdrücken können und ob Personen Medien anhand spezifischer Kriterien auswählen. Außerdem wird die Bereitschaft zum Lernen und Reflektieren im Umgang mit digitalen Medien erfasst.

- Selbstführungskompetenz: inwiefern sich Personen bei remote Arbeit selbst strukturieren und ihre Zeit managen können.

- Einschätzung der positiven Auswirkungen digitaler Zusammenarbeit

- Informationsüberlastung

- Zufriedenheit mit der digitalen Arbeit

- Wohlbefinden/Stress

Auf Technikebene:

- Digitalisierungsgrad: Ausmaß an digitaler Kommunikation, des verteilten Arbeitens und digitaler Geschäftsprozesse.

- Medienausstattung: Verfügbarkeit von Hard- und Software zur Erledigung von Arbeitsaufgaben.

- Digitale Geschäftsprozesse: inwieweit diese nachvollziehbar und sinnvoll umgesetzt sind, als nützlich bewertet werden und das Potenzial ausschöpfen.

- Nützlichkeit der digitalen Medien: wie zuverlässig/nützlich die vorhandenen digitalen Medien sind.

Auf Organisationsebene:

- IT-Support: angebotene Unterstützung zur Nutzung digitaler Medien.

- Führung: digitales Führungsverhalten (Kommunikation, Monitoring und Verhalten)

- Bedingungen, Regeln und Regeleinhaltung digitaler Zusammenarbeit

- Erreichbarkeitserwartungen an Beschäftigte

- Zeitdruck

- Effektivität digitaler Arbeit

- Identifikation mit dem Unternehmen

Diese Aspekte können in einer Kurzversion mit 67 Fragen erfasst werden (Dauer ca. 15-20 min). Alternativ können einzelne Aspekte vertieft abgefragt werden (Langversion mit insgesamt 105 Fragen, Dauer ca. $30 \mathrm{~min}$ ). 


\subsubsection{TeamCheck}

Die Ergebnisse unserer Umfragen bei Führungskräften und ihrer Teammitglieder der Projekt- und Umsetzungspartner zu Anforderungen, Belastungsschwerpunkten und Ressourcen digitaler Führungs- und Teamarbeit zeigen, dass die Koordination in und Führung von digitalen Teams spezifische Anforderungen stellt. Um effektiv zusammenarbeiten zu können, müssen Teammitglieder und -führung nicht nur ein gemeinsames Verständnis dessen haben, was erreicht werden soll und wer was bis wann zu tun hat (sog. im Team geteilte mentale Modelle, TMM), sondern sich auch einig sein, welche Medien sich für welche Zwecke, in welchen Situationen eignen, und auf welche Art und Weise sie diese bei der digitalen Zusammenarbeit nutzen, vgl. [2].

Der TeamCheck als online Feedbacktool zur Unterstützung digitaler Teamkoordination und -führung greift diese Erkenntnisse auf. Er ermöglicht online zu erfassen, inwieweit Teammitglieder bei der digitalen Arbeit gemeinsame Vorstellungen arbeitsrelevanter Themen haben, inwieweit sich Informationsbedarfe oder Überlastungen abzeichnen und visualisiert diese mithilfe eines graphischen Anzeigesystems, um Reflexions- und Regulationsprozesse im Team anzustoßen.

Zentrale Bausteine des TeamChecks sind, inwieweit Teamführung und -mitglieder zu folgenden Aspekten der digitalen Teamarbeit gemeinsame Vorstellungen haben, vgl. [9]:

- Aspekte der Aufgabe: Status Quo der Teamziele, Vorgehensweise zur Erreichung der Ziele und Überblick über anstehende Aufgaben (Aufgabenbezogene TMM);

- Aspekte des Teams: Fähigkeiten und Kompetenzen der Teammitglieder sowie deren Verantwortlichkeiten und Rollen (Teambezogene TMM);

- Aspekte der Zeit: Deadlines, die Dauer der Aufgaben und die verfügbare Zeit für diese Aufgaben (Zeitbezogene TMM);

- Aspekte der Medien: welche Medien im Team für welchen Zweck verwendet werden und die Art und Weise, wie Medien im Team genutzt werden (Medienbezogenes TMM);

- Aspekte der Situation: Bewusstsein über situative Anforderungen (Situationsbezogenes TMM).

Neben diesen Kernmodulen können noch folgende weitere Aspekte von der Teamführung ausgewählt und zur Beurteilung virtueller Teammeetings eingesetzt werden:

- Vertrauen im Team: ob die Teammitglieder sich bezüglich ihrer Arbeit und Einhaltung von Absprachen aufeinander verlassen können.

- Offene Teamkommunikation: ob die Teammitglieder vertrauensvoll über Schwierigkeiten sprechen und Rat einfordern können.

- Gemeinsames Lernen: ob die Teammitglieder zusammen über neue Lösungen nachdenken und sich gegenseitig unterstützen.

- Wissensaustausch: ob das Team sich regelmäßig zu relevanten Kenntnissen und Wissen austauscht.

- Auslastung: ob Teammitglieder unter Zeitdruck stehen, parallel Aufgaben bearbeiten müssen oder häufig unterbrochen werden. 
- Informationsprozesse: ob jedem Teammitglied relevante Informationen zur Aufgabenerledigung vorliegen.

- Reflexion von Prozessen: ob das Team über Strategien, den aktuellen Stand und die Koordination der Zusammenarbeit diskutiert.

- Reflexion von Ergebnissen: ob das Team Arbeitsergebnisse, Lerngewinn und den Beitrag der Teamleistung zum Unternehmen bespricht.

- Bewertung eines Meetings: ob das Meeting nützlich und relevant für die Teammitglieder war, ob alles Wichtige besprochen wurde und ob sie aufmerksam zugehört haben.

- Identifikation mit dem Team: inwieweit die Teammitglieder sich mit dem Team identifizieren.

Der TeamCheck erlaubt es, eine Umfrage zur Evaluation eines virtuellen Meetings am Ende dieses Meetings in Minutenschnelle durchzuführen und die Ergebnisse gleich einzusehen. So kann die Teamführung diese direkt mit ihrem Team besprechen und sich abzeichnende Unklarheiten sofort aufdecken. Bei der Nutzung des TeamChecks als online Feedbacktool werden die Ergebnisse entsprechend des vereinbarten Detailgrades automatisiert angezeigt. Es können drei Abstufungen gewählt werden: „gering“, „,mittel“ und „hoch“. „Gering“ empfiehlt sich z. B. bei sehr kleinen Teams, die Ergebnisbalken sind dann entweder komplett rot, gelb oder grün eingefärbt. Sobald mindestens ein Teammitglied der Aussage gar nicht oder nicht zustimmt, wird der Balken Rot angezeigt. Bei „mittel“ sind die Balken in rot, gelb und grün, bei „hoch“ in je zwei rot-, gelb- und grün-Farbtönen eingefärbt und spiegeln jeweils das Verhältnis des Antwortverhaltens der Teammitglieder dar, siehe Abb. 13.2. Zusätzlich steht eine Ergebnisrückmeldung zum Download zur Verfügung. Dieser Report enthält zusätzliche Reflexionsfragen und Handlungsempfehlungen. Im nächsten Teammeeting können dann die schon durchdachten Themen gemeinsam reflektiert und so ein konstruktiver und lösungsorientierter Austausch angeregt werden.

Die Teammitglieder der Pilotgruppen, die den TeamCheck erprobten, fanden ihn mehrheitlich nützlich, um Meetings zu bewerten und den Austausch über Teamprozesse

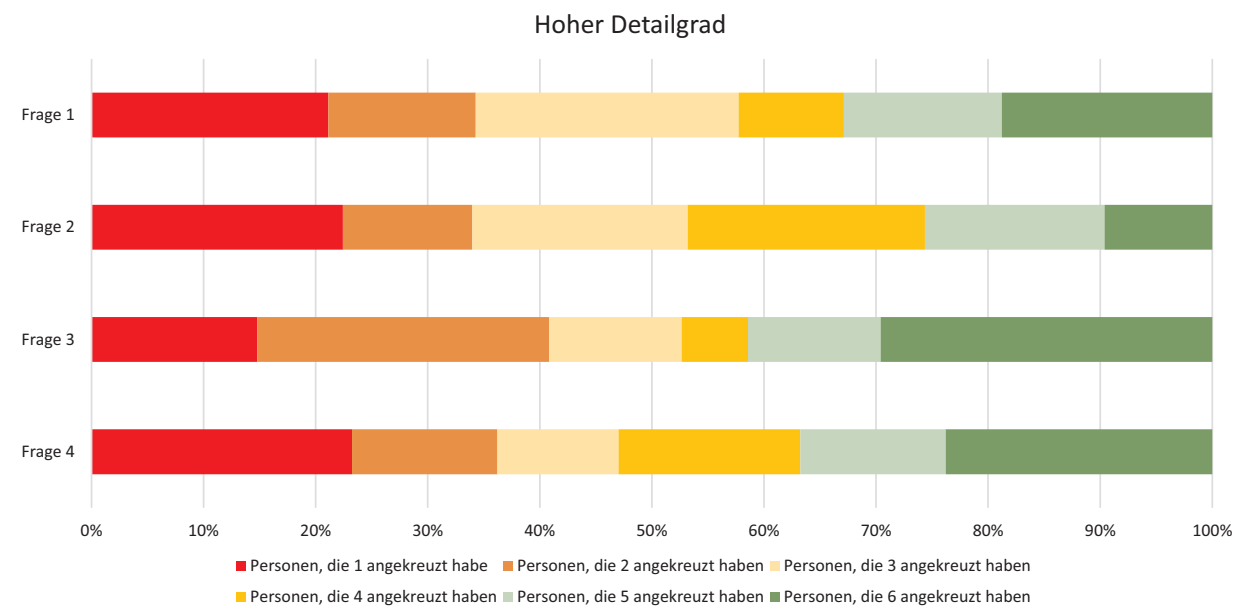

Abb. 13.2 Beispielhafte Ergebnisdarstellung im TeamCheck bei hohem Detailgrad 
sowie konkrete Maßnahmen zur Verbesserung der Teamarbeit anzuregen. Dieser Austausch hat in den Pilotgruppen in Teilen schon zur Umsetzung konkreter Maßnahmen geführt, die die Effizienz und Leistung des Teams erhöhen sollen. Die Mehrheit beurteilte die Fragen des Teamchecks als verständlich und sieht alle wichtigen Aspekte einer erfolgreichen digitalen Teamarbeit abgedeckt.

\subsubsection{IdeaCheck}

Für den IdeaCheck ergab die Anforderungsanalyse als zentrale Gestaltungsziele: Transparenz des Innovationsprozesses, Nachvollziehbarkeit der Umsetzungsentscheidungen hinsichtlich Innovationen, Effektivität des Innovationsprozesses (Ideen sind erfolgsversprechend und mit den vorhandenen zeitlichen und finanziellen Ressourcen umsetzbar) und Sicherung der Ideen (Ideenspeicher).

Um diese Ziele zu erreichen orientiert sich der IdeaCheck an der Stage-Gate-Struktur der Produktentwicklung, vgl. [4]. Eine Idee muss mehrere Phasen (Stages) durchlaufen, bis sie angenommen wird. Die Phasen sind durch Bewertungsentscheidungen (Gates) getrennt, bei denen anhand einer Scorecard geprüft wird, ob eine Idee die Kriterien erfüllt, um von einer Phase in die nächste zu kommen, siehe Abb. 13.3. Sowohl die Phasenanzahl als auch die relevanten Kriterien für die Umsetzungsentscheidung können verändert werden, um eine unternehmensspezifische Anpassung zu ermöglichen. Zusätzlich ist es möglich, Kategorien anzulegen, beispielsweise für Produkte oder Unternehmensbereiche (z. B. Entwicklung) und jeweils kategorienspezifische Phasen und Gates zu konfigurieren. Ideen derselben Kategorie werden anhand derselben Kriterien (z. B. strategische Bedeutung) bewertet und durchlaufen dieselbe Anzahl an Schritten bis zur Umsetzungsentscheidung. Bewertungskriterien können an den verschiedenen Gates bereichs- und unternehmensspezifisch festgelegt und gewichtet werden, um die jeweiligen Unternehmensziele abzubilden.

Der IdeaCheck bietet ein zentrales digitales Sammelbecken für Innovationsideen. Alle Beschäftigten haben die Gelegenheit, Ideen einzubringen. Nach dem Anlegen einer Idee kann diese als Entwurf gespeichert werden. Dies ermöglicht es, eine Idee schnell festzuhalten und zu einem späteren Zeitpunkt zu vervollständigen. Bevor eine Idee im Tool veröffentlicht wird, kann keine andere Person die Idee einsehen. Nach der Veröffentlichung

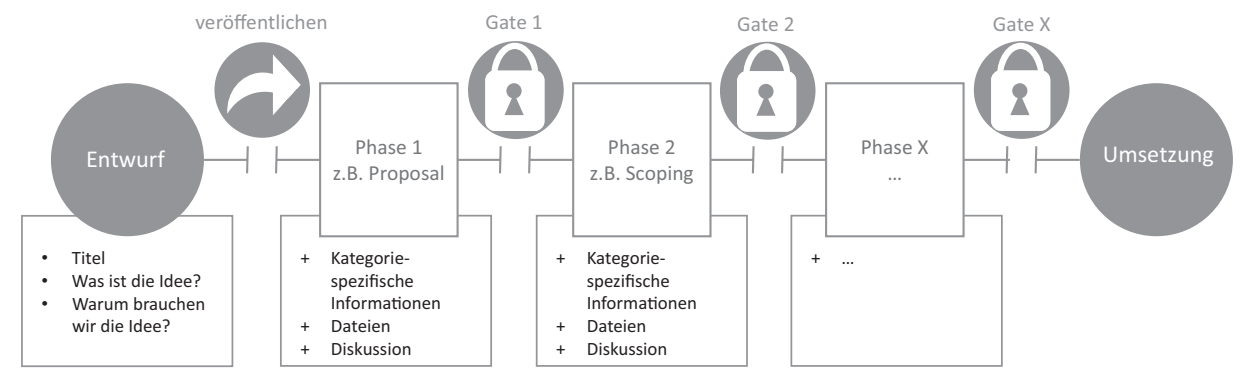

Abb. 13.3 Schematische Darstellung des Ideenbewertungsprozesses im IdeaCheck 
ist es möglich, sich an den Ideen anderer zu beteiligen. Man kann sich in Form von Diskussionsbeiträgen einbringen, eine Idee mit der eigenen Expertise unterstützen und einer Idee folgen und damit über Neuigkeiten auf dem Laufenden bleiben. Im Tool ist für jeden Nutzenden ein Profil mit Informationen zur Abteilungszugehörigkeit hinterlegt, welches zusätzlich um Informationen zu Expertise und Interessen ergänzt werden kann. Diese Funktionen ermöglichen es, aktiv nach Unterstützenden mit bestimmten Kenntnissen zu suchen, um die Idee weiterzuentwickeln. Ist eine Idee zur Bewertung freigegeben, bewertet ein Entscheidungsgremium die Idee anhand der Scorecard. Mitglieder des Gremiums sind in der Regel Führungskräfte oder Fachkräfte mit entsprechenden Kenntnissen und Befugnissen. Wer diesem Entscheidungsgremium angehört, kann unternehmensspezifisch festgelegt werden. Nachdem alle Mitglieder des Gremiums eine Idee bewertet und ihre Entscheidung ggfs. begründet haben, erhalten die Personen, die sie eingereicht haben, ein Feedback. Erreicht eine Idee die benötigte Punktzahl bei der Bewertung, geht sie in die nächste Phase, andernfalls verbleibt sie in der aktuellen Phase und man kann auf Grundlage des Feedbacks entscheiden, ob man an der Idee weiterarbeiten und diese anschließend erneut zur Bewertung freigeben oder die Idee nicht weiterverfolgen will. Das Entscheidungsgremium ist dazu angehalten, eine entsprechende Empfehlung zu geben. Die Ergebnisse der Nutzerumfrage des IdeaChecks zeigen, dass sie die Benutzeroberfläche positiv wahrnehmen und das Tool als intuitiv bedienbar bewerten, siehe Abb. 13.4.

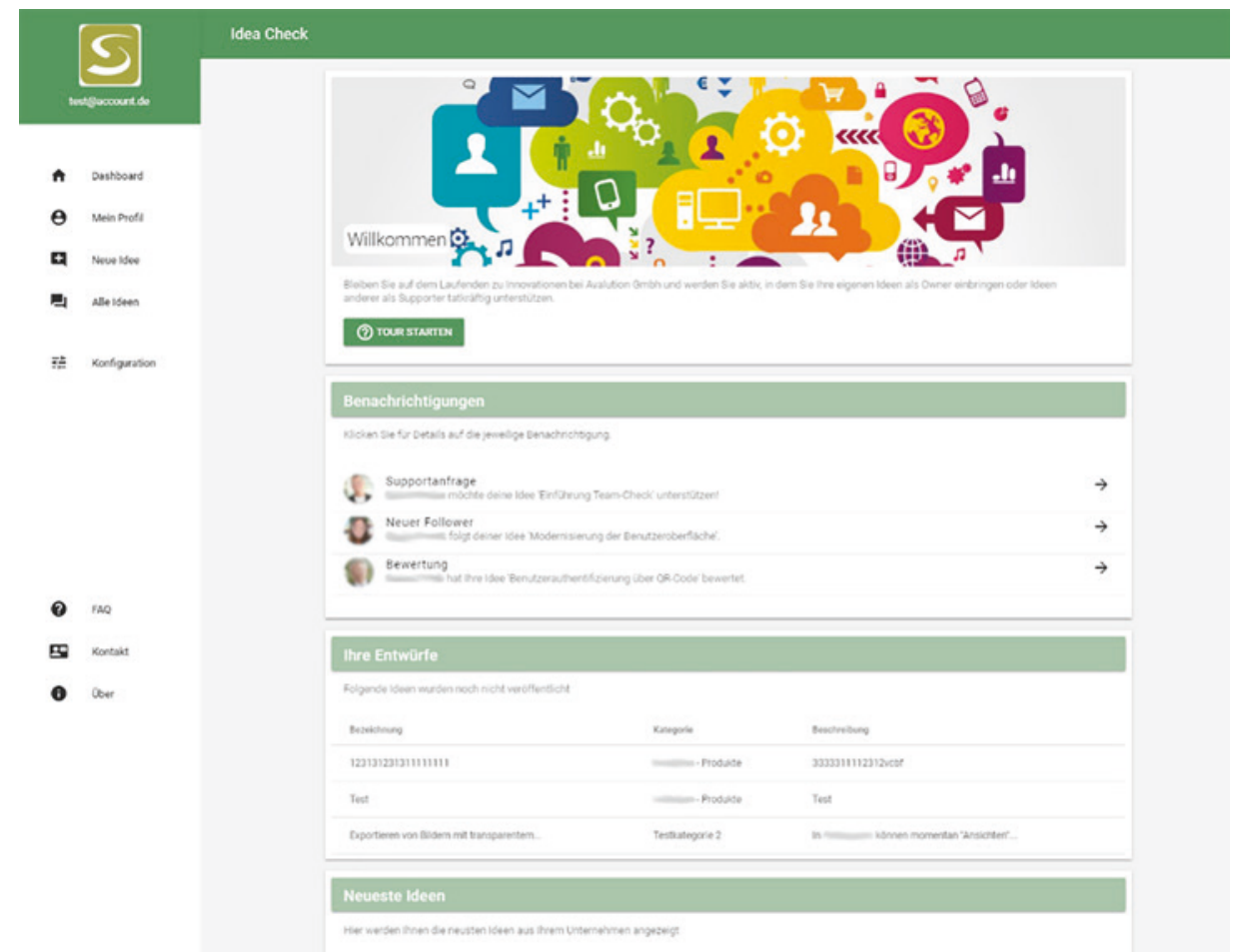

Abb. 13.4 Dashboard im IdeaCheck 
Die Nutzerumfrage zeigt, dass der IdeaCheck das Potenzial hat, den Innovationsprozess transparenter und für alle einsehbar $\mathrm{zu}$ machen sowie einen übersichtlichen Ideenspeicher zu bieten. Insbesondere die Möglichkeit Ideen anderer mit der eigenen Expertise zu unterstützen wird positiv bewertet, vgl. [1, 11].

\subsubsection{Ressourcenstärkende Führung}

Der im vLead-Teilprojekt des IAQ entwickelte Screening-Fragebogen dient zur Identifikation der individuellen Belastungen und Ressourcen der opFK. Die teilnehmenden opFK arbeiteten in großem Umfang virtuell mit Teammitgliedern unterschiedlicher Kulturen in unterschiedlichen Zeitzonen, die z.T. weltweit verteilt sind. Ihr organisatorisches Umfeld war sehr dynamisch, geprägt von Restrukturierungsmaßnahmen, Teamveränderungen und häufig wechselnden Aufgabenprioritäten und Zielen. Als Belastungen nannten die opFk am häufigsten Arbeitsunterbrechungen, Entgrenzung, Aneignungsbehinderungen, Zusatzaufwand und Zeitdruck. Diese Belastungen stimmen mit allgemeinen Befunden aus dem IT-Bereich überein, vgl. [6]. Sie sind u. a. eine Konsequenz der kommunikationsintensiven Tätigkeit der opFk.

Zur Entwicklung der individuellen Bewältigungsstrategien der opFK wurde ein individuelles Beratungsvorgehen im Rahmen von Feedback-Gesprächen verfolgt. Dieses orientiert sich am Vorgehen der Stressmanagement-Intervention [3]. Zur Identifikation der individuellen Belastungen und Ressourcen wird zu Beginn der Gespräche das Screening-Instrument ausgefüllt. Die konkrete Arbeitssituation wird dann reflektiert und darauf aufbauend konkrete Verbesserungen entwickelt sowie Hinweise zu Handlungsmöglichkeiten vermittelt. In einem Follow-Up werden dann Erfahrungen und Umsetzungsschwierigkeiten bearbeitet. Dieses Vorgehen ermöglicht die Arbeits- und Regenerationssituation zu reflektieren und neue Handlungsmöglichkeiten und Gestaltungsansätze im Gespräch zu entwickeln.

Entsprechend der oben beschriebenen Belastungen lag ein Schwerpunkt der im Gespräch entwickelten Maßnahmen in der Nutzung von Blockzeiten, um besonders bei konzeptionellen Arbeiten Arbeitsunterbrechungen entgegenzuwirken, vgl. [5]. Beim Verhältnis von Verausgabung und Entspannung gab es ebenfalls Potenzial bei vielen Teilnehmenden. Erarbeitete Maßnahmen waren die regelmäßige Nutzung von Kurzpausen sowie ein Arbeiten analog der eigenen Leistungskurve, um die Leistungsfähigkeit über den Tag zu erhalten. Ergänzt wurde dies in einigen Fällen durch Vorschläge für ein Abschlussritual, vgl. [10] - z. B. die tägliche Tasse Tee nach der Ankunft zuhause - und den bewussten Übergang zu Feierabend und Familienzeit, um einerseits mögliche Entgrenzung zu reduzieren und zum anderen die Regeneration zu fördern. 


\subsubsection{Zeitkompetenz und Vertrauen in virtuellen Führungsstrukturen}

Als Ergebnis des vLead-Teilprojekts des iap wurde der Kompass - Zeit und Vertrauen gemeinsam mit den Umsetzungspartnern erarbeitet und erprobt. Er umfasst die Analyse von Situationen und von Gesprächen zur Arbeitsweise von Teams, zur Förderung von gegenseitigem Verständnis, von Nachvollziehbarkeit und Transparenz. Ferner werden konkrete Maßnahmen zur Förderung virtueller Teamarbeit, mit zahlreichen und vielfältigen praktischen Handlungsempfehlungen, Checklisten sowie Umsetzungsbeispielen beschrieben. Der Kompass - Zeit und Vertrauen enthält komplementäre Lösungsansätze, die durch den modularen Aufbau individuell angepasst werden können. Die Selektion von Lösungsansätzen kann nach verschiedenen Faktoren, etwa dem Anwendenden (z. B. Führungskraft, Team oder Organisation) oder dem gewünschten Outcome erfolgen. Dies ermöglicht eine situationsgerechte Implementierung. Dabei sollten die Vorschläge jedoch nicht als Musterlösung interpretiert werden, sondern lediglich als Impuls oder Hinweis.

Der Kompass - Zeit und Vertrauen umfasst folgende fünf zentrale Instrumente: E-Talk-Unsere Sprache, E-Leadership-Kompetenz, Dashboard, Vereinbarung - Zeit und Vertrauen, Overload - Wahrnehmung und Bewältigung von Überlast.

- Der E-Talk gibt Teams Anregungen zur Diskussion der Kommunikationsstrukturen im Team. Er enthält Leitfragen, um die Reflexion der Kommunikation anzuregen, zentrale Leitsätze für virtuelle Kommunikation und Checklisten für virtuelle Meetings.

- Das Instrument E-Leadership-Kompetenz richtet sich an Personen, die in Teams eine Leitungs- oder Koordinierungsfunktion übernehmen: Führungskräfte, Teamleitungen und Personen, die in Teilen Führungsaufgaben innehaben. Sie können mit dem Instrument ihre aktuell vorhandenen Stärken und Entwicklungsfelder identifizieren. Zudem gibt es für Teamleitungen zu dem jeweils identifizierten Handlungsfeld passende Gestaltungstipps. Die vier Handlungsfelder sind: Beziehung zu Mitarbeitenden, Kontrolle \& Autonomie, Koordination \& Orientierung und Individuelle Entwicklung der Mitarbeitenden.

- Das Dashboard liefert, ähnlich wie ein Cockpit, im Optimalfall eine Übersicht über alle Aufgaben auf Team-Ebene sowie Informationen zum Umgang mit Zeit auf individueller Ebene und gibt hierzu einen Überblick über zentrale Zeitplantechniken.

- Mit der Vereinbarung - Zeit \& Vertrauen können Teams die teaminternen Kommunikationsregeln verbindlich festhalten, die sie sich im E-Talk überlegt 
haben. Die Vereinbarung gibt eine Orientierungshilfe für den Umgang und die Kommunikation im Team.

- Das Instrument Overload - Wahrnehmung und Bewältigung von Überlast - gibt Betroffenen sowie deren Kolleginnen und Kollegen Informationen zu hoher Arbeitsbelastung sowie Hilfestellungen zur Einordnung und Empfehlungen für den Umgang mit der jeweiligen Situation.

Darüber hinaus gibt es mit E-Coffee, E-Daily und Ready to fly Anregungen zur konkreten Gestaltung von virtueller Teamarbeit.

- E-Coffee gibt Mitarbeitenden an unterschiedlicher Standorten Tipps zur Organisation und Durchführung informeller persönlicher Gespräche per Video analog einer Begegnung in der Kaffeeküche.

- E-Daily gibt Tipps zum regelmäßigen arbeitsbezogenen Austausch von Informationen, um Klarheit und Orientierung über Aufgaben und deren Zweck herbeizuführen.

- Ready to fly ist eine Checkliste mit Tipps für die Vorbereitung und Moderation virtueller Meetings.

In einem iterativen Evaluierungsprozess haben die Umsetzungspartner die Empfehlungen des Handbuchs in der Praxis angewendet und evaluiert. In den Pilotteams wurden erste positive Erfahrungen mit den Maßnahmen berichtet. Die Möglichkeit, die Instrumente nach individuellen Bedürfnissen anzupassen, wurde mehrfach positiv hervorgehoben. Als Ergebnis wurde eine höhere Zufriedenheit der Beschäftigten sowie eine effizientere Teamarbeit beschrieben, vgl. [8].

\subsection{Ausblick}

Die im Projekt vLead entwickelten Instrumente sollen bei weiteren Umsetzungspartnern auch anderer Branchen erprobt und dadurch ihr breiter Transfer gefördert werden.

\subsection{Hinweis auf Transfermaterialien}

Auf der Homepage des Projekts https://vlead.de stehen die im Projekt entwickelten Instrumente zur Nutzung sowie begleitende Handbücher als Download zur Verfügung. 


\section{Projektpartner und Aufgaben}

- Universität Trier, Arbeits-, Betriebs- und Organisationspsychologie (ABO) Digitale Projekt- und Teamarbeit leistungs- und ressourcenförderlich führen

- Universität Duisburg-Essen, Institut Arbeit und Qualifikation (IAQ) Ressourcenstärkende Führung - operative Führungskräfte in virtuellen Kontexten stärken und gesund erhalten

- FOM Hochschule für Oekonomie \& Management gGmbH, Institut für Arbeit \& Personal (iap)

Zeitkompetenz und Vertrauen in virtuellen Führungsstrukturen

- Human Solutions Verwaltungs GmbH

Entwicklung des vLead Innovationsprozess Tools IdeaCheck zur Unterstützung digitaler Führung teamübergreifender Innovationsprozesse

- Data One GmbH

Entwicklung des vLead Belastungs-Screening OrgaCheck und Prozessmonitoring-Tools TeamCheck zur effektiven, ressourcenförderlichen digitalen Führung

\section{Literatur}

1. Antoni CH, Röltgen AT, Bernardy V, Müller R (2020) Entwicklung und Evaluation der Auswirkungen eines digitalen Softwaretools zur Unterstützung kollaborativer Innovationsprozesse. In: Gesellschaft für Arbeitswissenschaft e. V. (Hrsg) Bericht zum 66. Arbeitswissenschaftlichen Kongress der Gesellschaft für Arbeitswissenschaft e.V. vom 16. bis 18. März 2020, Berlin, Digitale Arbeit, digitaler Wandel, digitaler Mensch? Beitrag A.9.1. GfA Press, Dortmund

2. Bernardy V, Müller R, Röltgen AT, Antoni CH (2019) Entwicklung eines Instruments zur Unterstützung der Bildung geteilter mentaler Modelle in digitalen Teams. In: Gesellschaft für Arbeitswissenschaft e. V. (Hrsg) Bericht zum 65. Arbeitswissenschaftlichen Kongress der Gesellschaft für Arbeitswissenschaft e.V. vom 27.02. bis 01.03.2019, Dresden, Arbeit interdisziplinär analysieren - bewerten - gestalten, Beitrag C.6.1. GfA Press, Dortmund

3. Busch C, Steinmetz B (2002) Stressmanagement und Führungskräfte. Gruppe. Interaktion. Organisation. Zeitschrift für Angewandte Organisationspsychologie (GIO) 33(4):385-401

4. Cooper RG (2014) What's next?: After stage-gate. Res Technol Manage 57(1):20-31

5. Gerlmaier A (2019) Blockzeiten für störungsfreies Arbeiten. In: Gerlmaier A, Latniak E (Hrsg) Handbuch psycho-soziale Gestaltung digitaler Produktionsarbeit. Springer Gabler, Wiesbaden, S 325-328

6. Gerlmaier A (2011) Stress und Burnout bei IT-Fachleuten - auf der Suche nach Ursachen. In: Gerlmaier A, Latniak E (Hrsg) Burnout in der IT-Branche: Ursachen und betriebliche Prävention. Asanger, Kröning, S 53-90

7. Hellert U, Müller F, Mander R (2018) Zeitkompetenz, Vertrauen und Prozessfeedback im Virtual Work Resource Model. In: der Zukunft A (Hrsg) Hermeier B Heupel T FichtnerRosada. Springer Gabler, Wiesbaden, S 145-161

8. Mander R, Hellert U, Müller F (2019) Gestaltungsansätze in virtuellen Kooperationsstrukturen: Zeitkompetenz und Vertrauen. In: Gesellschaft für Arbeitswissenschaft e. V. (Hrsg) 
Bericht zum 65. Arbeitswissenschaftlichen Kongress der Gesellschaft für Arbeitswissenschaft e.V. vom 27.02. bis 01.03.2019, Dresden, Arbeit interdisziplinär analysieren - bewerten gestalten, Beitrag C.6.3. GfA Press, Dortmund

9. Müller R, Antoni CH (2019) Einflussfaktoren und Auswirkungen eines gemeinsamen Medienverständnisses in virtuellen Teams. Gruppe. Interaktion. Organisation. Zeitschrift für Angewandte Organisationspsychologie (GIO) 50(1):25-32. https://doi.org/10.1007/s11612019-00447-3

10. Reif JAM, Spieß E (2018) Erholung. In: Reif JAM, Spieß E, Stadler P (Hrsg) Effektiver Umgang mit Stress: Gesundheitsmanagement im Beruf. Springer, Berlin, Heidelberg, S 131138

11. Röltgen AT, Bernardy V, Müller R, Antoni CH (2020) Entwicklung, Einsatz und Evaluation eines Tools für digitales Ideenmanagement. Ein Fallbeispiel. Gruppe. Interaktion. Zeitschrift für Angewandte Organisationspsychologie Organisation (GIO) 51:49-58. https://doi. org/10.1007/s11612-020-00500-6 . https://rdcu.be/b0TRM

12. Venkatesh V, Morris MG, Davis GB, Davis FD (2003) User acceptance of information technology: toward a unified view. MIS Q 27(3):425-478. https://doi.org/10.2307/30036540

Open Access Dieses Kapitel wird unter der Creative Commons Namensnennung 4.0 International Lizenz (http://creativecommons.org/licenses/by/4.0/deed.de) veröffentlicht, welche die Nutzung, Vervielfältigung, Bearbeitung, Verbreitung und Wiedergabe in jeglichem Medium und Format erlaubt, sofern Sie den/die ursprünglichen Autor(en) und die Quelle ordnungsgemäß nennen, einen Link zur Creative Commons Lizenz beifügen und angeben, ob Änderungen vorgenommen wurden.

Die in diesem Kapitel enthaltenen Bilder und sonstiges Drittmaterial unterliegen ebenfalls der genannten Creative Commons Lizenz, sofern sich aus der Abbildungslegende nichts anderes ergibt. Sofern das betreffende Material nicht unter der genannten Creative Commons Lizenz steht und die betreffende Handlung nicht nach gesetzlichen Vorschriften erlaubt ist, ist für die oben aufgeführten Weiterverwendungen des Materials die Einwilligung des jeweiligen Rechteinhabers einzuholen.

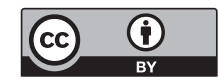

